\title{
Interval-Valued Neutrosophic Soft Rough Sets
}

\author{
Said Broumi ${ }^{1}$ and Flornetin Smarandache ${ }^{2}$ \\ ${ }^{1}$ Faculty of Lettres and Humanities, University of Hassan II-Casablanca, Hay El Baraka Ben M'sik, BP 7951, Casablanca, Morocco \\ ${ }^{2}$ Department of Mathematics, University of New Mexico, 705 Gurley Avenue, Gallup, NM 87301, USA
}

Correspondence should be addressed to Said Broumi; broumisaid78@gmail.com

Received 23 June 2014; Revised 17 November 2014; Accepted 11 December 2014

Academic Editor: Neeraj Mittal

Copyright (C) 2015 S. Broumi and F. Smarandache. This is an open access article distributed under the Creative Commons Attribution License, which permits unrestricted use, distribution, and reproduction in any medium, provided the original work is properly cited.

We first defined interval-valued neutrosophic soft rough sets (IVN-soft rough sets for short) which combine interval-valued neutrosophic soft set and rough sets and studied some of its basic properties. This concept is an extension of interval-valued intuitionistic fuzzy soft rough sets (IVIF-soft rough sets).

\section{Introduction}

In 1999, Smarandache introduced the theory of neutrosophic set (NS) [1], which is the generalization of the classical sets, conventional fuzzy set [2], intuitionistic fuzzy set [3], interval-valued fuzzy set [4], and so on. The concept of neutrosophic set handles indeterminate data whereas fuzzy set theory and intuitionistic fuzzy set theory failed when the relation is indeterminate.

Recently, works on the neutrosophic set theory is progressing rapidly. Bhowmik and Pal $[5,6]$ defined "intuitionistic neutrosophic set." Later on Salama and Alblowi [7] introduced another concept called "generalized neutrosophic set." Wang et al. [8] proposed another extension of neutrosophic set which is "single valued neutrosophic sets." Also, Wang et al. [9] introduced the notion of interval-valued neutrosophic sets (IVNSs) which is an instance of neutrosophic set. The IVNSs are characterized by an interval membership degree, interval indeterminacy degree, and interval nonmembership degree. Georgiev [10] explored some properties of the neutrosophic logic and proposed a general simplification of the neutrosophic sets into a subclass of theirs, comprised of elements of $R^{3}$. Ye $[11,12]$ defined similarity measures between interval neutrosophic sets and their multicriteria decision making method. Majumdar and Samant [13] proposed some types of similarity and entropy of neutrosophic sets. Broumi and Smarandache [14-16] proposed several similarity measures of neutrosophic sets. Chi and Peide [17] extended TOPSIS to interval neutrosophic sets and so on.

In 1999, a Russian researcher, Molodotsov, proposed a new mathematical tool called "soft set theory" [18], for dealing with uncertainty and how soft set theory is free from the parameterization inadequacy syndrome of fuzzy set theory, rough set theory, and probability theory. Recently, Deli [19] introduced the concept of interval-valued neutrosophic soft set as a combination of interval neutrosophic set and soft set. This concept generalizes the concept of the soft set, fuzzy soft set [20], intuitionistic fuzzy soft set [21], interval-valued intuitionistic fuzzy soft set [22], neutrosophic soft set, and intuitionistic neutrosophic soft set [23].

The rough set theory that was introduced by Pawlak [24] in 1982, which is a technique for managing the uncertainty and imperfection, can analyze incomplete information effectively. Therefore, many models have been built upon different aspect, that is, universe, relations, object, and operators by many scholars [25-30] such as rough fuzzy sets, fuzzy rough sets, generalized fuzzy rough set, rough intuitionistic fuzzy set, and intuitionistic fuzzy rough sets [31]. It has been successfully applied in many fields such as attribute reduction [32-35], feature selection [36-38], and rule extraction [3942]. The rough sets theory approximates any subset of objects of the universe by two sets, called the lower and upper approximations. It focuses on the ambiguity caused by the limited discernibility of objects in the universe of discourse. 
Moreover, many new rough set models have also been established by combining the Pawlak rough set with other uncertainty theories such as soft set theory. Feng [43] provided a framework to combine fuzzy sets, rough sets, and soft sets all together, which gives rise to several interesting new concepts such as rough soft sets, soft rough sets, and soft rough fuzzy sets. The combination of hybrid structures of soft sets and rough sets models was also discussed by some researchers [44-46]. Later on, Zhang et al. [47] proposed the notions of soft rough intuitionistic fuzzy sets and intuitionistic fuzzy soft rough sets, which can be seen as two new generalized soft rough set models, and investigated some properties of soft rough intuitionistic fuzzy sets and intuitionistic fuzzy soft rough sets in detail. Also Saha and Mukherjee [48] proposed the concept of the notion of soft interval-valued intuitionistic fuzzy rough sets.

More recently, Broumi et al. [49] combined neutrosophic sets with rough sets in a new hybrid mathematical structure called "rough neutrosophic sets" handling incomplete and indeterminate information. The concept of rough neutrosophic sets generalizes fuzzy rough sets and intuitionistic fuzzy rough sets. Also Salama and Broumi [50] studied roughness of neutrosophic sets. Based on the equivalence relation on the universe of discourse, Mukherjee et al. [51] introduced soft lower and upper approximation of interval-valued intuitionistic fuzzy set in Pawlak's approximation space. Motivated by the idea of interval-valued intuitionistic fuzzy soft rough sets introduced in [52], we extend the interval intuitionistic fuzzy lower and upper approximations to the case of an intervalvalued neutrosophic set. The concept of an interval-valued neutrosophic soft rough set is introduced by coupling both the interval-valued neutrosophic soft sets and rough sets.

The paper is structured as follows. In Section 2, we first recall the necessary background on rough sets and interval neutrosophic soft set. Section 3 reviews various proposals for the definition of interval neutrosophic soft rough sets and examines their respective properties. Section 4 presents a multiciteria group decision making scheme under interval-valued neutrosophic soft rough set. Section 5 presents an application of multiciteria group decision making scheme regarding the candidate selection problem. Finally we conclude the paper.

\section{Preliminaries}

Throughout this paper, let $U$ be a universal set and let $E$ be the set of all possible parameters under consideration with respect to $U$; usually parameters are attributes, characteristics, or properties of objects in $U$. We now recall some basic notions of soft sets, interval neutrosophic sets, neutrosophic soft set, interval neutrosophic soft set, rough set, and rough neutrosophic sets. For more details the reader may refer to $[1,9,18,19,24,49]$.

Definition 1. Let $U$ be a universe of discourse; then the neutrosophic set $A$ is an object having the form $A=\left\{\left\langle x: \mu_{A}(x)\right.\right.$, $\left.\left.v_{A}(x), \omega_{A}(x)\right\rangle, x \in U\right\}$, where the functions $\mu_{A}(x), v_{A}(x)$, and $\left.\omega_{A}(x): U \rightarrow\right]^{-} 0,1^{+}$[ define, respectively, the degree of membership, the degree of indeterminacy, and the degree of nonmembership of the element $x \in X$ to the set $A$ with the condition

$$
{ }^{-} 0 \leq \sup \mu_{A}(x)+\sup \nu_{A}(x)+\sup \omega_{A}(x) \leq 3^{+} .
$$

From philosophical point of view, the neutrosophic set takes the value from real standard or nonstandard subsets of ]$^{-} 0,1^{+}[\text {. So instead of }]^{-} 0,1^{+}$[ we need to take the interval $[0,1]$ for technical applications because $]^{-} 0,1^{+}[$will be difficult to apply in the real applications such as in scientific and engineering problems.

Definition 2 (see [1]). Let $X$ be a space of points (objects) with generic elements in $X$ denoted by $x$. An interval-valued neutrosophic set (for short IVNS) $A$ in $X$ is characterized by truth-membership function $\mu_{A}(x)$, indeterminacymembership function $v_{A}(x)$, and falsity-membership function $\omega_{A}(x)$. For each point $x$ in $X$, one has that $\mu_{A}(x), v_{A}(x)$, and $\omega_{A}(x) \in \operatorname{int}([0,1])$.

For two IVNSs,

$$
\begin{aligned}
A_{\mathrm{IVNS}}=\left\{\left\langlex,\left[\mu_{A}^{L}(x), \mu_{A}^{U}(x)\right],\right.\right. \\
\left.\left.\quad\left[\nu_{A}^{L}(x), \nu_{A}^{U}(x)\right],\left[\omega_{A}^{L}(x), \omega_{A}^{U}(x)\right]\right\rangle \mid x \in X\right\} .
\end{aligned}
$$

And $B_{\mathrm{IVNS}}=\left\{\left\langle x,\left[\mu_{B}^{L}(x), \mu_{B}^{U}(x)\right],\left[\nu_{B}^{L}(x), v_{B}^{U}(x)\right],\left[\omega_{B}^{L}(x)\right.\right.\right.$, $\left.\left.\left.\omega_{B}^{U}(x)\right]\right\rangle \mid x \in X\right\}$; the two relations are defined as follows:

(1) $A_{\text {IVNS }} \subseteq B_{\text {IVNS }}$ if and only if $\mu_{A}^{L}(x) \leq \mu_{B}^{L}(x), \mu_{A}^{U}(x) \leq$ $\mu_{B}^{U}(x), \nu_{A}^{L}(x) \geq v_{B}^{L}(x), \omega_{A}^{U}(x) \geq \omega_{B}^{U}(x), \omega_{A}^{L}(x) \geq$ $\omega_{B}^{L}(x)$, and $\omega_{A}^{U}(x) \geq \omega_{B}^{U}(x)$

(2) $A_{\text {IVNS }}=B_{\text {IVNS }}$ if and only if $\mu_{A}(x)=\mu_{B}(x), v_{A}(x)=$ $\nu_{B}(x)$, and $\omega_{A}(x)=\omega_{B}(x)$ for any $x \in X$.

The complement of $A_{\text {IVNS }}$ is denoted by $A_{\text {IVNS }}^{o}$ and is defined by

$$
\begin{gathered}
A_{\mathrm{IVNS}}^{o}=\left\{\left\langlex,\left[\omega_{A}^{L}(x), \omega_{A}^{U}(x)\right],\left[1-v_{A}^{U}(x), 1-v_{A}^{L}(x)\right],\right.\right. \\
\left.\left.\left[\mu_{A}^{L}(x), \mu_{A}^{U}(x)\right]\right\rangle \mid x \in X\right\}
\end{gathered}
$$

$A \cap B$

$$
\begin{aligned}
& =\left\{\left\langlex,\left[\min \left(\mu_{A}^{L}(x), \mu_{B}^{L}(x)\right), \min \left(\mu_{A}^{U}(x), \mu_{B}^{U}(x)\right)\right],\right.\right. \\
& \quad\left[\max \left(v_{A}^{L}(x), v_{B}^{L}(x)\right), \max \left(v_{A}^{U}(x), v_{B}^{U}(x)\right)\right], \\
& \left.\quad\left[\max \left(\omega_{A}^{L}(x), \omega_{B}^{L}(x)\right), \max \left(\omega_{A}^{U}(x), \omega_{B}^{U}(x)\right)\right]\right\rangle: \\
& x \in X\}
\end{aligned}
$$

$A \cup B$

$$
\begin{aligned}
=\left\{\left\langlex,\left[\max \left(\mu_{A}^{L}(x), \mu_{B}^{L}(x)\right), \max \left(\mu_{A}^{U}(x), \mu_{B}^{U}(x)\right)\right],\right.\right. \\
\quad\left[\min \left(v_{A}^{L}(x), v_{B}^{L}(x)\right), \min \left(v_{A}^{U}(x), v_{B}^{U}(x)\right)\right], \\
{\left.\left[\min \left(\omega_{A}^{L}(x), \omega_{B}^{L}(x)\right), \min \left(\omega_{A}^{U}(x), \omega_{B}^{U}(x)\right)\right]\right\rangle: } \\
x \in X\} .
\end{aligned}
$$


As an illustration, let us consider the following example.

Example 3. Assume that the universe of discourse $U=\left\{x_{1}\right.$, $\left.x_{2}, x_{3}\right\}$, where $x_{1}$ characterizes the capability, $x_{2}$ characterizes the trustworthiness, and $x_{3}$ indicates the prices of the objects. It may be further assumed that the values of $x_{1}, x_{2}$, and $x_{3}$ are in $[0,1]$ and they are obtained from some questionnaires of some experts. The experts may impose their opinion in three components, namely, the degree of goodness, the degree of indeterminacy, and the degree of poorness to explain the characteristics of the objects. Suppose $A$ is an interval-valued neutrosophic set (IVNS) of $U$, such that $A=\left\{\left\langle x_{1},[0.3,0.4]\right.\right.$, $[0.5,0.6],[0.4,0.5]\rangle,\left\langle x_{2},[0.1,0.2],[0.3,0.4],[0.6,0.7]\right\rangle,\left\langle x_{3}\right.$, $[0.2,0.4],[0.4,0.5],[0.4,0.6]\rangle\}$, where the degree of goodness of capability is $[0.3,0.4]$, degree of indeterminacy of capability is $[0.5,0.6]$, degree of falsity of capability is $[0.4,0.5]$, and so forth.

Definition 4 (see [18]). Let $U$ be an initial universe set and let $E$ be a set of parameters. Let $P(U)$ denote the power set of $U$. Consider a nonempty set $A, A \subset E$. A pair $(K, A)$ is called a soft set over $U$, where $K$ is a mapping given by $K: A \rightarrow P(U)$.

As an illustration, let us consider the following example.

Example 5. Suppose that $U$ is the set of houses under consideration; say $U=\left\{h_{1}, h_{2}, \ldots, h_{5}\right\}$. Let $E$ be the set of some attributes of such houses; say $E=\left\{e_{1}, e_{2}, \ldots, e_{8}\right\}$, where $e_{1}$, $e_{2}, \ldots, e_{8}$ stand for the attributes "beautiful," "costly," "green surroundings," and "moderate," respectively.

In this case, to define a soft set means to point out expensive houses, beautiful houses, and so on. For example, the soft set $(K, A)$ that describes the "attractiveness of the houses" in the opinion of a buyer, say Thomas, may be defined as follows:

$$
\begin{gathered}
A=\left\{e_{1}, e_{2}, e_{3}, e_{4}, e_{5}\right\} ; \\
K\left(e_{1}\right)=\left\{h_{2}, h_{3}, h_{5}\right\}, \quad K\left(e_{2}\right)=\left\{h_{2}, h_{4}\right\}, \\
K\left(e_{3}\right)=\left\{h_{1}\right\}, \quad K\left(e_{4}\right)=U, \quad K\left(e_{5}\right)=\left\{h_{3}, h_{5}\right\} .
\end{gathered}
$$

Definition 6 (see [19]). Let $U$ be an initial universe set and let $A \subset E$ be a set of parameters. Let IVNS $(U)$ denote the set of all interval neutrosophic subsets of $U$. The collection $(K, A)$ is termed to be the soft interval neutrosophic set over $U$, where $F$ is a mapping given by $K: A \rightarrow \operatorname{IVNS}(U)$.

The interval neutrosophic soft set defined over a universe is denoted by INSS.

Here,

(1) $\Upsilon$ is an ivn-soft subset of $\Psi$, denoted by $\Upsilon \Subset \Psi$, if $K(e) \subseteq L(e)$ for all $e \in E$;

(2) $\Upsilon$ is an ivn-soft equal to $\Psi$, denoted by $\Upsilon=\Psi$, if $K(e)=L(e)$ for all $e \in E$;

(3) the complement of $\Upsilon$ is denoted by $\Upsilon^{c}$ and is defined by $\Upsilon^{c}=\left\{\left(x, K^{o}(x)\right): x \in E\right\} ;$

(4) the union of $\Upsilon$ and $\Psi$ is denoted by $\Upsilon \cup^{\prime \prime} \Psi$, if $K(e) \cup$ $L(e)$ for all $e \in E$;

(5) the intersection of $\Upsilon$ and $\Psi$ is denoted by $\Upsilon \cap^{\prime \prime} \Psi$, if $K(e) \cup L(e)$ for all $e \in E$.
Example 7. Let $U$ be the set of houses under consideration and $E$ is the set of parameters (or qualities). Each parameter is an interval neutrosophic word or sentence involving interval neutrosophic words. Consider $E=\{$ beautiful, costly, moderate, expensive $\}$. In this case, to define an interval neutrosophic soft set means to point out beautiful houses, costly houses, and so on. Suppose that there are four houses in the universe $U$ given by $U=\left\{h_{1}, h_{2}, h_{3}, h_{4}\right\}$ and the set of parameters $A=\left\{e_{1}, e_{2}, e_{3}\right\}$, where each $e_{i}$ is a specific criterion for houses:

$$
\begin{aligned}
& e_{1} \text { stands for "beautiful," } \\
& e_{2} \text { stands for "costly," } \\
& e_{3} \text { stands for "moderate." }
\end{aligned}
$$

Suppose that

$$
\begin{aligned}
& K(\text { beautiful })=\left\{\left\langle h_{1},[0.5,0.6],[0.6,0.7],[0.3,0.4]\right\rangle,\right. \\
&\left\langle h_{2},[0.4,0.5],[0.7,0.8],[0.2,0.3]\right\rangle, \\
&\left\langle h_{3},[0.6,0.7],[0.2,0.3],[0.3,0.5]\right\rangle, \\
&\left.\left\langle h_{4},[0.7,0.8],[0.3,0.4],[0.2,0.4]\right\rangle\right\} . \\
& K(\text { costly })=\left\{\left\langle h_{1},[0.3,0.6],[0.2,0.7],[0.1,0.4]\right\rangle,\right.\left\langle h_{2},[0.3,0.5],[0.6,0.8],[0.2,0.6]\right\rangle, \\
&\left\langle h_{3},[0.3,0.7],[0.1,0.3],[0.3,0.6]\right\rangle, \\
&\left.\left\langle h_{4},[0.6,0.8],[0.2,0.4],[0.2,0.5]\right\rangle\right\} . \\
& K(\text { moderate })=\left\{\left\langle h_{1},[0.5,0.8],[0.4,0.7],[0.3,0.6]\right\rangle,\right.\left\langle h_{2},[0.3,0.5],[0.7,0.9],[0.2,0.4]\right\rangle, \\
&\left\langle h_{3},[0.1,0.7],[0.3,0.3],[0.3,0.6]\right\rangle, \\
&\left.\left\langle h_{4},[0.3,0.8],[0.2,0.4],[0.3,0.6]\right\rangle\right\} .
\end{aligned}
$$

Definition 8 (see [24]). Let $R$ be an equivalence relation on the universal set $U$. Then the pair $(U, R)$ is called a Pawlak approximation space. An equivalence class of $R$ containing $x$ will be denoted by $[x]_{R}$. Now for $X \subseteq U$, the lower and upper approximation of $X$ with respect to $(U, R)$ are denoted by, respectively, $R_{*} X$ and $R^{*} X$ and are defined by

$$
\begin{gathered}
R_{*} X=\left\{x \in U:[x]_{R} \subseteq X\right\}, \\
R^{*} X=\left\{x \in U:[x]_{R} \cap X \neq \emptyset\right\} .
\end{gathered}
$$

Now if $R^{*} X=R_{*} X$, then $X$ is called definable; otherwise $X$ is called a rough set.

Definition 9 (see [49]). Let $U$ be a nonnull set and let $R$ be an equivalence relation on $U$. Let $F$ be neutrosophic set in $U$ with the membership function $\mu_{F}$, indeterminacy function $\nu_{F}$, and nonmembership function $\omega_{F}$. Then the lower and upper 
rough approximations of $F$ in $(U, R)$ are denoted by $\underline{R}(F)$ and $\bar{R}(F)$ and, respectively, defined as follows:

$$
\begin{aligned}
& \bar{R}(F)=\left\{\left\langle x, \mu_{\bar{R}(F)}(x), \nu_{\bar{R}(F)}(x), \omega_{\bar{R}(F)}(x)\right\rangle \mid x \in U\right\}, \\
& \underline{R}(F)=\left\{\left\langle x, \mu_{\underline{R}(F)}(x), \nu_{\underline{R}(F)}(x), \omega_{\underline{R}(F)}(x)\right\rangle \mid x \in U\right\},
\end{aligned}
$$

where

$$
\begin{array}{ll}
\mu_{\bar{R}(F)}(x)=\bigvee_{y \in[x]_{R}} \mu_{F}(y), & \nu_{\bar{R}(F)}(x)=\bigwedge_{y \in[x]_{R}} \nu_{F}(y), \\
\omega_{\bar{R}(F)}(x)=\bigwedge_{y \in[x]_{R}} \omega_{F}(y), & \mu_{\underline{R}(F)}(x)=\bigwedge_{y \in[x]_{R}} \mu_{F}(y), \\
\nu_{\underline{R}(F)}(x)=\bigvee_{y \in[x]_{R}} \nu_{F}(y), & \omega_{\underline{R}(F)}(x)=\bigvee_{y \in[x]_{R}} \omega_{F}(y) .
\end{array}
$$

It is easy to observe that $\bar{R}(F)$ and $\underline{R}(F)$ are two neutrosophic sets in $U$; thus NS mapping $\bar{R}, R: R(U) \rightarrow R(U)$ are, respectively, referred to as the upper and lower rough NS approximation operators, and the pair $(\underline{R}(F), \bar{R}(F))$ is called the rough neutrosophic set.

\section{Interval Neutrosophic Soft Rough Set}

This section is an attempt to extend the concept of an intervalvalued intuitionistic fuzzy soft rough set [52] to the case of an interval-valued neutrosophic rough set.

Definition 10. Let $\Theta=(f, A)$ be full soft set over $U$ and let the pair $S=(U, \Theta)$ be the soft approximation space. Then for an interval-valued neutrosophic set $\sigma \in$ IVNS $^{U}$, the lower and upper soft rough approximations of $\sigma$ with respect to $S$ are denoted by $\underline{N}_{S}(\sigma)$ and $\bar{N}_{S}(\sigma)$, respectively, which are intervalvalued neutrosophic sets in $U$ given by

$$
\begin{gathered}
\underline{N}_{S}(\sigma)=\left\{\left\langlex,\left[\bigwedge\left\{\inf \mu_{\sigma}(y): \exists a \in A(\{x, y\} \subseteq f(a))\right\},\right.\right.\right. \\
\bigwedge\left\{\sup \mu_{\sigma}(y):\right. \\
\exists a \in A(\{x, y\} \subseteq f(a))\}], \\
{\left[\bigvee\left\{\inf \nu_{\sigma}(y): \exists a \in A(\{x, y\} \subseteq f(a))\right\},\right.} \\
\\
\left.\bigvee\left\{\sup v_{\sigma}(y): \exists a \in A(\{x, y\} \subseteq f(a))\right\}\right], \\
{\left[\bigvee\left\{\inf \omega_{\sigma}(y): \exists a \in A(\{x, y\} \subseteq f(a))\right\},\right.} \\
\\
\left.\left.\bigvee\left\{\sup \omega_{\sigma}(y): \exists a \in A(\{x, y\} \subseteq f(a))\right\}\right]\right\rangle: \\
x \in U\}
\end{gathered}
$$

$$
\begin{aligned}
& \bar{N}_{S}(\sigma)=\{\langle x, \backslash \bigvee\left\{\inf \mu_{\sigma}(y): \exists a \in A(\{x, y\} \subseteq f(a))\right\}, \\
&\left.\bigvee\left\{\sup \mu_{\sigma}(y): \exists a \in A(\{x, y\} \subseteq f(a))\right\}\right], \\
& \bigwedge\left\{\inf \nu_{\sigma}(y): \exists a \in A(\{x, y\} \subseteq f(a))\right\}, \\
&\left.\bigwedge\left\{\sup \nu_{\sigma}(y): \exists a \in A(\{x, y\} \subseteq f(a))\right\}\right], \\
& \bigwedge\left\{\inf \omega_{\sigma}(y): \exists a \in A(\{x, y\} \subseteq f(a))\right\}, \\
&\left.\left.\bigwedge\left\{\sup \omega_{\sigma}(y): \exists a \in A(\{x, y\} \subseteq f(a))\right\}\right]\right\rangle: \\
&x \in U\} .
\end{aligned}
$$

The operators $\underline{N}_{S}(\sigma)$ and $\bar{N}_{S}(\sigma)$ are called the lower and upper soft rough approximation operators on interval-valued neutrosophic sets. If $\underline{N}_{S}(\sigma)=\bar{N}_{S}(\sigma)$, then $\sigma$ is said to be soft interval-valued neutrosophic definable; otherwise it is called an interval-valued neutrosophic soft rough set.

Theorem 11. Let $\Theta=(f, A)$ be a full soft set over $U$ and let $S=$ $(U, \Theta)$ be the soft approximation space. Then for $\sigma \in I V N S^{U}$ one has

(i) $\underline{N}_{S}(\sigma) \quad=\quad\left\{\left\langle x,\left[\bigwedge_{x \in f(a)} \bigwedge_{y \in f(a)} \inf \mu_{\sigma}(y)\right.\right.\right.$, $\left.\bigwedge_{x \in f(a)} \bigwedge_{y \in f(a)} \sup \mu_{\sigma}(y)\right],\left[\bigvee_{x \in f(a)} \bigvee_{y \in f(a)} \inf \nu_{\sigma}(y)\right.$ $\left.\bigvee_{x \in f(a)} \bigvee_{y \in f(a)} \sup v_{\sigma}(y)\right],\left[\bigvee_{x \in f(a)} \bigvee_{y \in f(a)} \inf \omega_{\sigma}(y)\right.$, $\left.\left.\bigvee_{x \in f(a)} \bigvee_{y \in f(a)} \sup \omega_{\sigma}(y)\right]\right\rangle: x \in U$;

(ii) $\bar{N}_{S}(\sigma) \quad=\quad\left\{\left\langle x,\left[\bigvee_{x \in f(a)} \bigvee_{y \in f(a)} \inf \mu_{\sigma}(y)\right.\right.\right.$, $\left.\bigvee_{x \in f(a)} \bigvee_{y \in f(a)} \sup \mu_{\sigma}(y)\right],\left[\bigwedge_{x \in f(a)} \bigwedge_{y \in f(a)} \inf \nu_{\sigma}(y)\right.$, $\left.\bigwedge_{x \in f(a)} \bigwedge_{y \in f(a)} \sup v_{\sigma}(y)\right],\left[\bigwedge_{x \in f(a)} \bigwedge_{y \in f(a)} \inf \omega_{\sigma}(y)\right.$, $\left.\left.\left.\bigwedge_{x \in f(a)} \bigwedge_{y \in f(a)} \sup \omega_{\sigma}(y)\right]\right\rangle: x \in U\right\}$.

Proof. (i) Let $a \in A$ and $x \in f(a)$. Then for $y \in f(a)$, we have $\{x, y\} \subseteq f(a)$ and hence inf $\mu_{\sigma}(y) \geq \bigwedge\left\{\inf \mu_{\sigma}(z): \exists a \in\right.$ $A\{x, z\} \subseteq f(a)\}$. Consequently,

$$
\bigwedge_{y \in f(a)} \inf \mu_{\sigma}(y) \geq \bigwedge\left\{\inf \mu_{\sigma}(z): \exists a \in A(\{x, z\} \subseteq f(a))\right\} .
$$

And so

$$
\begin{aligned}
& \bigwedge_{x \in f(a)} \bigwedge_{y \in f(a)} \inf \mu_{\sigma}(y) \\
& \geq \bigwedge\left\{\inf \mu_{\sigma}(z): \exists a \in A(\{x, z\} \subseteq f(a))\right\} .
\end{aligned}
$$

Similarly, it can be shown that

$$
\bigwedge_{x \in f(a)} \bigwedge_{y \in f(a)} \sup \mu_{\sigma}(y)
$$

$$
\geq \bigwedge\left\{\sup \mu_{\sigma}(z): \exists a \in A(\{x, z\} \subseteq f(a))\right\} .
$$


Thus, we get

$$
\begin{aligned}
& {\left[\bigwedge\left\{\inf \mu_{\sigma}(z): \exists a \in A(\{x, z\} \subseteq f(a))\right\}\right.} \\
& \left.\bigwedge\left\{\sup \mu_{\sigma}(z): \exists a \in A(\{x, z\} \subseteq f(a))\right\}\right] \\
& \quad \subseteq\left[\bigwedge_{x \in f(a)} \bigwedge_{y \in f(a)} \inf \mu_{\sigma}(y), \bigwedge_{x \in f(a)} \bigwedge_{y \in f(a)} \sup \mu_{\sigma}(y)\right] .
\end{aligned}
$$

In a similar manner it can be shown that

$$
\begin{aligned}
& {\left[\bigvee\left\{\inf \nu_{\sigma}(z): \exists \underline{N}_{S}(\sigma)\right\}\right.} \\
& \left.\bigvee\left\{\sup \nu_{\sigma}(z): \exists a \in A(\{x, z\} \subseteq f(a))\right\}\right] \\
& \quad \subseteq\left[\bigvee_{x \in f(a)} \bigvee_{y \in f(a)} \inf \nu_{\sigma}(y), \bigvee_{x \in f(a)} \bigvee_{y \in f(a)} \sup \nu_{\sigma}(y)\right]
\end{aligned}
$$

In a similar manner it can be shown that

$$
\begin{aligned}
& {\left[\bigvee_{x \in f(a)} \bigvee_{y \in f(a)} \inf \omega_{\sigma}(y), \bigvee_{x \in f(a)} \bigvee_{y \in f(a)} \sup \omega_{\sigma}(y)\right] } \\
& \times {\left[\bigvee\left\{\inf \omega_{\sigma}(z): \exists a \in A(\{x, z\} \subseteq f(a))\right\},\right.} \\
&\left.\bigvee\left\{\sup \omega_{\sigma}(z): \exists a \in A(\{x, z\} \subseteq f(a))\right\}\right] \\
& \subseteq {\left[\bigvee_{x \in f(a)} \bigvee_{y \in f(a)} \inf \omega_{\sigma}(y), \bigvee_{x \in f(a)} \bigvee_{y \in f(a)} \sup \omega_{\sigma}(y)\right] . }
\end{aligned}
$$

From (14), (15), and (16) we observe that

$\underline{N}_{S}(\sigma)$

$$
\begin{gathered}
\subseteq\left\{\left[\bigwedge_{x \in f(a)} \bigwedge_{y \in f(a)} \inf \mu_{\sigma}(y), \bigwedge_{x \in f(a)} \bigwedge_{y \in f(a)} \sup \mu_{\sigma}(y)\right],\right. \\
{\left[\bigvee_{x \in f(a)} \bigvee_{y \in f(a)} \inf \nu_{\sigma}(y), \bigvee_{x \in f(a)} \bigvee_{y \in f(a)} \sup \nu_{\sigma}(y)\right],} \\
\left.\left[\bigvee_{x \in f(a)} \bigvee_{y \in f(a)} \inf \omega_{\sigma}(y), \bigvee_{x \in f(a)} \bigvee_{y \in f(a)} \sup \omega_{\sigma}(y)\right]\right\} .
\end{gathered}
$$

Now we prove that

$$
\begin{aligned}
& \left\{\left[\bigwedge_{x \in f(a)} \bigwedge_{y \in f(a)} \inf \mu_{\sigma}(y), \bigwedge_{x \in f(a)} \bigwedge_{y \in f(a)} \sup \mu_{\sigma}(y)\right],\right. \\
& {\left[\bigvee_{x \in f(a)} \bigvee_{y \in f(a)} \inf \nu_{\sigma}(y), \bigvee_{x \in f(a)} \bigvee_{y \in f(a)} \sup \nu_{\sigma}(y)\right],} \\
& {\left[\subseteq \underline{N}_{S}(\sigma) .\right.}
\end{aligned}
$$

Let us suppose that $a \in A$ such that $\{x, z\} \subseteq f(a)$. Then $a \in$ $f(a), z \in f(a)$, and hence

$$
\inf \mu_{\sigma}(z) \geq \bigwedge_{x \in f(a)} \bigwedge_{y \in f(a)} \inf \mu_{\sigma}(y) .
$$

Consequently,

$$
\begin{aligned}
& \bigwedge\left\{\inf \mu_{\sigma}(z): \exists a \in A(\{x, z\} \subseteq f(a))\right\} \\
& \geq \bigwedge_{x \in f(a)} \bigwedge_{y \in f(a)} \inf \mu_{\sigma}(y) .
\end{aligned}
$$

Similarly, it can be shown that

$$
\begin{aligned}
\bigwedge & \left\{\sup \mu_{\sigma}(z): \exists a \in A(\{x, z\} \subseteq f(a))\right\} \\
\geq & \bigwedge_{x \in f(a)} \bigwedge_{y \in f(a)} \sup \mu_{\sigma}(y) .
\end{aligned}
$$

Thus we get

$$
\begin{gathered}
{\left[\bigwedge_{x \in f(a)} \bigwedge_{y \in f(a)} \inf \mu_{\sigma}(y), \bigwedge_{x \in f(a)} \bigwedge_{y \in f(a)} \sup \mu_{\sigma}(y)\right]} \\
\subseteq\left[\bigwedge\left\{\inf \mu_{\sigma}(z): \exists a \in A(\{x, z\} \subseteq f(a))\right\},\right. \\
\left.\bigwedge\left\{\sup \mu_{\sigma}(z): \exists a \in A(\{x, z\} \subseteq f(a))\right\}\right] .
\end{gathered}
$$

In a similar manner it can be shown that

$$
\begin{gathered}
{\left[\bigvee_{x \in f(a)} \bigvee_{y \in f(a)} \inf v_{\sigma}(y), \bigvee_{x \in f(a)} \bigvee_{y \in f(a)} \sup v_{\sigma}(y)\right]} \\
\subseteq\left[\bigvee\left\{\inf \nu_{\sigma}(z): \exists a \in A(\{x, z\} \subseteq f(a))\right\},\right. \\
\left.\bigvee\left\{\sup v_{\sigma}(z): \exists a \in A(\{x, z\} \subseteq f(a))\right\}\right] .
\end{gathered}
$$

In a similar manner it can be shown that

$$
\begin{gathered}
{\left[\bigvee_{x \in f(a)} \bigvee_{y \in f(a)} \inf \omega_{\sigma}(y), \bigvee_{x \in f(a)} \bigvee_{y \in f(a)} \sup \omega_{\sigma}(y)\right]} \\
\subseteq\left[\bigvee\left\{\inf \omega_{\sigma}(z): \exists a \in A(\{x, z\} \subseteq f(a))\right\},\right. \\
\left.\bigvee\left\{\sup \omega_{\sigma}(z): \exists a \in A(\{x, z\} \subseteq f(a))\right\}\right] .
\end{gathered}
$$


From (22), (23), and (24) we observe that

$$
\begin{aligned}
& \left\{\left[\bigwedge_{x \in f(a)} \bigwedge_{y \in f(a)} \inf \mu_{\sigma}(y), \bigwedge_{x \in f(a)} \bigwedge_{y \in f(a)} \sup \mu_{\sigma}(y)\right],\right. \\
& {\left[\bigvee_{x \in f(a)} \bigvee_{y \in f(a)} \inf v_{\sigma}(y), \bigvee_{x \in f(a)} \bigvee_{y \in f(a)} \sup v_{\sigma}(y)\right],} \\
& \left.\left[\bigvee_{x \in f(a)} \bigvee_{y \in f(a)} \inf \omega_{\sigma}(y), \bigvee_{x \in f(a)} \bigvee_{y \in f(a)} \sup \omega_{\sigma}(y)\right]\right\} \\
& \subseteq \underline{N}_{S}(\sigma) .
\end{aligned}
$$

From (17) and (25), we have

$$
\begin{aligned}
\underline{N}_{S}(\sigma) & \left\{\left\langlex,\left[\bigwedge_{x \in f(a)} \bigwedge_{y \in f(a)} \inf \mu_{\sigma}(y), \bigwedge_{x \in f(a)} \bigwedge_{y \in f(a)} \sup \mu_{\sigma}(y)\right],\right.\right. \\
& {\left[\bigvee_{x \in f(a)} \bigvee_{y \in f(a)} \inf \nu_{\sigma}(y), \bigvee_{x \in f(a)} \bigvee_{y \in f(a)} \sup \nu_{\sigma}(y)\right], } \\
& {\left[\bigvee_{x \in f(a)} \bigvee_{y \in f(a)} \inf \omega_{\sigma}(y),\right.} \\
& \left.\left.\left.\bigvee_{x \in f(a)} \bigvee_{y \in f(a)} \sup \omega_{\sigma}(y)\right]\right\rangle: x \in U\right\} .
\end{aligned}
$$

(ii) Proof is similar to that in (i).

Theorem 12. Let $\Theta=(f, A)$ be a full soft set over $U$ and let $S=(U, \Theta)$ be the soft approximation space. Then for $\sigma, \lambda \in$ IVNS ${ }^{U}$ one has

(1) $\underline{N}_{s}(\emptyset)=\emptyset=\bar{N}_{s}(\emptyset)$;

(2) $\underline{N}_{s}(U)=U=\bar{N}_{s}(U)$;

(3) $\sigma \subseteq \lambda \Rightarrow \underline{N}(\sigma) \subseteq \underline{N}(\lambda)$;

(4) $\sigma \subseteq \lambda \Rightarrow \bar{N}_{s}(\sigma) \subseteq \bar{N}_{s}(\lambda)$;

(5) $\underline{N}_{s}(\sigma \cap \lambda) \subseteq \underline{N}_{s}(\sigma) \cap \underline{N}_{s}(\lambda)$;

(6) $\bar{N}_{s}(\sigma \cap \lambda) \subseteq \bar{N}_{s}(\sigma) \cap \bar{N}_{s}(\lambda)$;

(7) $\underline{N}(\sigma) \cup \underline{N}(\lambda) \subseteq \underline{N}(\sigma \cup \lambda)$;

(8) $\bar{N}_{s}(\sigma) \cup \bar{N}_{s}(\lambda) \subseteq \bar{N}_{s}(\sigma \cup \lambda)$.
Proof. (1)-(4) are straightforward.

(5) We have

$$
\begin{gathered}
\sigma=\left\{\left\langlex,\left[\inf \mu_{\sigma}(x), \sup \mu_{\sigma}(x)\right],\right.\right. \\
{\left[\inf \nu_{\sigma}(x), \sup \nu_{\sigma}(x)\right],} \\
\left.\left.\left[\inf \omega_{\sigma}(x), \sup \omega_{\sigma}(x)\right]\right\rangle: x \in U\right\}, \\
\lambda=\left\{\left\langlex,\left[\inf \mu_{\lambda}(x), \sup \mu_{\lambda}(x)\right],\right.\right. \\
{\left[\inf \nu_{\lambda}(x), \sup \nu_{\lambda}(x)\right],} \\
\left.\left.\quad\left[\inf \omega_{\lambda}(x), \sup \omega_{\lambda}(x)\right]\right\rangle: x \in U\right\}, \\
\sigma \wedge \lambda=\left\{\left[\inf \mu_{\sigma \cap \lambda}(x), \sup \mu_{\sigma \cap \lambda}(x)\right],\right. \\
{\left[\inf \nu_{\sigma \cap \lambda}(x), \sup \nu_{\sigma \cap \lambda}(x)\right],} \\
\left.\left.\left[\inf \omega_{\sigma \cap \lambda}(x), \sup \omega_{\sigma \cap \lambda}(x)\right]\right\rangle: x \in U\right\} .
\end{gathered}
$$

Now

$\underline{N}_{s}(\sigma \cap \lambda)$

$=\left\{\left\langle x,\left[\bigwedge\left\{\inf \mu_{\sigma \cap \lambda}(y): \exists a \in A(\{x, z\} \subseteq f(a))\right\}\right.\right.\right.$, $\left.\bigwedge\left\{\sup \mu_{\sigma \cap \lambda}(y): \exists a \in A\{x, y\} \subseteq f(a)\right\}\right]$,

$\left[\bigvee\left\{\inf \nu_{\sigma \cap \lambda}(z): \exists a \in A(\{x, y\} \subseteq f(a))\right\}\right.$,

$\left.\bigvee\left\{\sup v_{\sigma \cap \lambda}(y): \exists a \in A(\{x, z\} \subseteq f(a))\right\}\right]$,

$\left[\bigvee\left\{\inf \omega_{\sigma \cap \lambda}(y): \exists a \in A(\{x, y\} \subseteq f(a))\right\}\right.$,

$\left.\left.\bigvee\left\{\sup \omega_{\sigma \cap \lambda}(y): \exists a \in A(\{x, y\} \subseteq f(a))\right\}\right]\right\rangle:$ $x \in U\}$

$=\left\{\left\langle x,\left[\bigwedge\left\{\min \left(\inf \mu_{\sigma}(y), \inf \mu_{\lambda}(y)\right):\right.\right.\right.\right.$

$\exists a \in A\{x, z\} \subseteq f(a)\}$,

$\bigwedge\left\{\min \left(\sup \mu_{\sigma}(y), \sup \mu_{\lambda}(y)\right):\right.$

$\exists a \in A(\{x, z\} \subseteq f(a))\}]$,

$\left\lceil\left\{\max \left(\inf \nu_{\sigma}(y), \inf \nu_{\lambda}(y)\right):\right.\right.$

$\exists a \in A(\{x, z\} \subseteq f(a))\}$,

$\bigvee\left\{\max \left(\sup v_{\sigma}(y), \sup v_{\lambda}(y)\right):\right.$

$\exists a \in A(\{x, z\} \subseteq f(a))\}]$,

$\left\lceil\left\{\max \left(\inf \omega_{\sigma}(y), \inf \omega_{\lambda}(y)\right):\right.\right.$

$\exists a \in A(\{x, z\} \subseteq f(a))\}$,

$\bigvee\left\{\max \left(\sup \omega_{\sigma}(y), \sup \omega_{\lambda}(y)\right):\right.$

$\exists a \in A(\{x, z\} \subseteq f(a))\}]\rangle: x \in U\}$. 
Since

$$
\begin{aligned}
& \min \left(\inf \mu_{\sigma}(y), \inf \mu_{\lambda}(y)\right) \leq \inf \mu_{\sigma}(y), \\
& \min \left(\inf \mu_{\sigma}(y), \inf \mu_{\lambda}(y)\right) \leq \inf \mu_{\lambda}(y)
\end{aligned}
$$

we have

$$
\begin{aligned}
& \bigwedge\left\{\min \left(\inf \mu_{\sigma}(y), \inf \mu_{\lambda}(y)\right): \exists a \in A(\{x, z\} \subseteq f(a))\right\} \\
& \leq \bigwedge\left\{\inf \mu_{\sigma}(y): \exists a \in A(\{x, z\} \subseteq f(a))\right\}, \\
& \bigwedge\left\{\min \left(\inf \mu_{\sigma}(y), \inf \mu_{\lambda}(y)\right): \exists a \in A(\{x, z\} \subseteq f(a))\right\} \\
& \leq \bigwedge\left\{\inf \mu_{\lambda}(y): \exists a \in A(\{x, z\} \subseteq f(a))\right\} .
\end{aligned}
$$

\section{Consequently,}

$$
\begin{gathered}
\bigwedge\left\{\min \left(\inf \mu_{\sigma}(y), \inf \mu_{\lambda}(y)\right): \exists a \in A(\{x, z\} \subseteq f(a))\right\} \\
\leq \min \left(\bigwedge\left\{\inf \mu_{\sigma}(y): \exists a \in A(\{x, z\} \subseteq f(a))\right\}\right. \\
\left.\bigwedge\left\{\inf \mu_{\lambda}(y): \exists a \in A(\{x, z\} \subseteq f(a))\right\}\right) .
\end{gathered}
$$

Similarly we can get

$$
\begin{gathered}
\bigwedge\left\{\min \left(\sup \mu_{\sigma}(y), \sup \mu_{\lambda}(y)\right): \exists a \in A\{x, y\} \subseteq f(a)\right\} \\
\leq \min \left(\bigwedge\left\{\sup \mu_{\sigma}(y): \exists a \in A(\{x, z\} \subseteq f(a))\right\},\right. \\
\left.\bigwedge\left\{\sup \mu_{\lambda}(y): \exists a \in A(\{x, z\} \subseteq f(a))\right\}\right) .
\end{gathered}
$$

Again since

$$
\begin{aligned}
& \max \left(\inf \nu_{\sigma}(y), \inf \nu_{\lambda}(y)\right) \geq \inf \nu_{\sigma}(y), \\
& \max \left(\inf \nu_{\sigma}(y), \inf \nu_{\lambda}(y)\right) \geq \inf \nu_{\lambda}(y),
\end{aligned}
$$

we have

$$
\begin{aligned}
& \bigvee\left\{\max \left(\inf \nu_{\sigma}(y), \inf \nu_{\lambda}(y)\right): \exists a \in A\{x, y\} \subseteq f(a)\right\} \\
& \geq \bigvee\left\{\inf \nu_{\sigma}(y): \exists a \in A(\{x, z\} \subseteq f(a))\right\}, \\
& \bigvee\left\{\max \left(\sup \nu_{\sigma}(y), \sup \nu_{\lambda}(y)\right): \exists a \in A\{x, y\} \subseteq f(a)\right\} \\
& \geq \bigvee\left\{\sup \nu_{\sigma}(y): \exists a \in A(\{x, z\} \subseteq f(a))\right\} .
\end{aligned}
$$

Consequently,

$$
\begin{gathered}
\bigvee\left\{\max \left(\inf \nu_{\sigma}(y), \inf \nu_{\lambda}(y)\right): \exists a \in A\{x, y\} \subseteq f(a)\right\} \\
\geq \max \left(\bigwedge\left\{\inf \nu_{\sigma}(y): \exists a \in A(\{x, z\} \subseteq f(a))\right\},\right. \\
\left.\bigwedge\left\{\inf \nu_{\lambda}(y): \exists a \in A\{x, y\} \subseteq f(a)\right\}\right) .
\end{gathered}
$$

Similarly we can get

$$
\begin{gathered}
\bigvee\left\{\max \left(\sup \nu_{\sigma}(y), \sup \nu_{\lambda}(y)\right): \exists a \in A\{x, y\} \subseteq f(a)\right\} \\
\geq \max \left(\bigwedge\left\{\sup v_{\sigma}(y): \exists a \in A(\{x, z\} \subseteq f(a))\right\}\right. \\
\left.\bigwedge\left\{\sup v_{\lambda}(y): \exists a \in A\{x, y\} \subseteq f(a)\right\}\right) .
\end{gathered}
$$

Again since

$$
\begin{aligned}
& \max \left(\inf \omega_{\sigma}(y), \inf \omega_{\lambda}(y)\right) \geq \inf \omega_{\sigma}(y), \\
& \max \left(\inf \omega_{\sigma}(y), \inf \omega_{\lambda}(y)\right) \geq \inf \omega_{\lambda}(y),
\end{aligned}
$$

we have

$$
\begin{aligned}
& \bigvee\left\{\max \left(\inf \omega_{\sigma}(y), \inf \omega_{\lambda}(y)\right): \exists a \in A\{x, y\} \subseteq f(a)\right\} \\
& \geq \bigvee\left\{\inf \omega_{\sigma}(y): \exists a \in A(\{x, z\} \subseteq f(a))\right\}, \\
& \bigvee\left\{\max \left(\sup \omega_{\sigma}(y), \sup \omega_{\lambda}(y)\right): \exists a \in A\{x, y\} \subseteq f(a)\right\} \\
& \geq \bigvee\left\{\sup \omega_{\sigma}(y): \exists a \in A(\{x, z\} \subseteq f(a))\right\} .
\end{aligned}
$$

Consequently,

$$
\begin{gathered}
\bigvee\left\{\max \left(\inf \omega_{\sigma}(y), \inf \omega_{\lambda}(y)\right): \exists a \in A\{x, y\} \subseteq f(a)\right\} \\
\geq \max \left(\bigwedge\left\{\inf \omega_{\sigma}(y): \exists a \in A(\{x, z\} \subseteq f(a))\right\}\right. \\
\left.\bigwedge\left\{\inf \omega_{\lambda}(y): \exists a \in A\{x, y\} \subseteq f(a)\right\}\right) .
\end{gathered}
$$

Similarly we can get

$$
\begin{gathered}
\bigvee\left\{\max \left(\sup \omega_{\sigma}(y), \sup \omega_{\lambda}(y)\right): \exists a \in A\{x, y\} \subseteq f(a)\right\} \\
\geq \max \left(\bigwedge\left\{\sup \omega_{\sigma}(y): \exists a \in A\{x, y\} \subseteq f(a)\right\},\right. \\
\left.\bigwedge\left\{\sup \omega_{\lambda}(y): \exists a \in A(\{x, z\} \subseteq f(a))\right\}\right) .
\end{gathered}
$$

Using, (31)-(40) we get from (28)

$$
\underline{N}_{s}(\sigma \cap \lambda) \subseteq \underline{N}_{s}(\sigma) \cap \underline{N}_{s}(\lambda) .
$$

(6) Proof is similar to (5). 
(7) We have

$$
\begin{gathered}
\sigma=\left\{\left\langlex,\left[\inf \mu_{\sigma}(x), \sup \mu_{\sigma}(x)\right],\right.\right. \\
{\left[\inf \nu_{\sigma}(x), \sup \nu_{\sigma}(x)\right],} \\
\left.\left.\left[\inf \omega_{\sigma}(x), \sup \omega_{\sigma}(x)\right]\right\rangle: x \in U\right\}, \\
\lambda=\left\{\left\langlex,\left[\inf \mu_{\lambda}(x), \sup \mu_{\lambda}(x)\right],\right.\right. \\
\quad\left[\inf \nu_{\lambda}(x), \sup v_{\lambda}(x)\right], \\
\left.\left.\left[\inf \omega_{\lambda}(x), \sup \omega_{\lambda}(x)\right]\right\rangle: x \in U\right\}, \\
\sigma \cup \lambda=\left\{\left\langlex,\left[\inf \mu_{\sigma \cup \lambda}(x), \sup \mu_{\sigma \cup \lambda}(x)\right],\right.\right. \\
{\left[\inf v_{\sigma \cup \lambda}(x), \sup v_{\sigma \cup \lambda}(x)\right],} \\
\left.\left.\left[\inf \omega_{\sigma \cup \lambda}(x), \sup \omega_{\sigma \cup \lambda}(x)\right]\right\rangle: x \in U\right\} .
\end{gathered}
$$

Now

$$
\begin{aligned}
\bar{N}_{s}(\sigma \cup \lambda) \\
=\left\{\left\langlex,\left[\bigwedge\left\{\inf \mu_{\sigma \cup \lambda}(y): \exists a \in A(\{x, y\} \subseteq f(a))\right\},\right.\right.\right. \\
\left.\bigwedge\left\{\sup \mu_{\sigma \cup \lambda}(y): \exists a \in A(\{x, z\} \subseteq f(a))\right\}\right],
\end{aligned}
$$$$
\left[\bigvee\left\{\inf \nu_{\sigma \cup \lambda}(z): \exists a \in A(\{x, y\} \subseteq f(a))\right\},\right.
$$$$
\left.\bigvee\left\{\sup v_{\sigma \cup \lambda}(y): \exists a \in A(\{x, y\} \subseteq f(a))\right\}\right],
$$$$
\left[\bigvee\left\{\inf \omega_{\sigma \cup \lambda}(y): \exists a \in A(\{x, y\} \subseteq f(a))\right\},\right.
$$$$
\left.\left.\bigvee\left\{\sup \omega_{\sigma \cup \lambda}(y): \exists a \in A(\{x, z\} \subseteq f(a))\right\}\right]\right\rangle:
$$$$
x \in U\}
$$$$
=\left\{\left\langlex,\left[\bigwedge \left\{\max \left(\inf \mu_{\sigma}(y), \inf \mu_{\lambda}(y)\right):\right.\right.\right.\right.
$$$$
\exists a \in A(\{x, z\} \subseteq f(a))\},
$$$$
\bigwedge\left\{\max \left(\sup \mu_{\sigma}(y), \sup \mu_{\lambda}(y)\right):\right.
$$$$
\exists a \in A(\{x, z\} \subseteq f(a))\}],
$$$$
\left[\bigvee \left\{\min \left(\inf \nu_{\sigma}(y), \inf \nu_{\lambda}(y)\right):\right.\right.
$$$$
\exists a \in A(\{x, z\} \subseteq f(a))\},
$$$$
\bigvee\left\{\min \left(\sup \nu_{\sigma}(y), \sup \nu_{\lambda}(y)\right):\right.
$$$$
\exists a \in A(\{x, z\} \subseteq f(a))\}],
$$$$
\left[\bigvee \left\{\min \left(\inf \omega_{\sigma}(y), \inf \omega_{\lambda}(y)\right):\right.\right.
$$$$
\exists a \in A(\{x, z\} \subseteq f(a))\},
$$$$
\bigvee\left\{\min \left(\sup \omega_{\sigma}(y), \sup \omega_{\lambda}(y)\right):\right.
$$$$
\exists a \in A(\{x, z\} \subseteq f(a))\}]\rangle: x \in U\} .
$$

Since

$$
\begin{aligned}
& \max \left(\inf \mu_{\sigma}(y), \inf \mu_{\lambda}(y)\right) \geq \inf \mu_{\sigma}(y), \\
& \max \left(\inf \mu_{\sigma}(y), \inf \mu_{\lambda}(y)\right) \geq \inf \mu_{\lambda}(y)
\end{aligned}
$$

we have

$$
\begin{aligned}
& \bigwedge\left\{\max \left(\inf \mu_{\sigma}(y), \inf \mu_{\lambda}(y)\right): \exists a \in A\{x, y\} \subseteq f(a)\right\} \\
& \geq \bigwedge\left\{\inf \mu_{\sigma}(y): \exists a \in A(\{x, z\} \subseteq f(a))\right\}, \\
& \bigwedge\left\{\max \left(\inf \mu_{\sigma}(y), \inf \mu_{\lambda}(y)\right): \exists a \in A\{x, y\} \subseteq f(a)\right\} \\
& \geq \bigwedge\left\{\inf \mu_{\lambda}(y): \exists a \in A(\{x, z\} \subseteq f(a))\right\} .
\end{aligned}
$$

Consequently,

$$
\begin{gathered}
\bigwedge\left\{\max \left(\inf \mu_{\sigma}(y), \inf \mu_{\lambda}(y)\right): \exists a \in A(\{x, y\} \subseteq f(a))\right\} \\
\geq \max \left(\bigwedge\left\{\inf \mu_{\sigma}(y): \exists a \in A(\{x, z\} \subseteq f(a))\right\},\right. \\
\left.\bigwedge\left\{\inf \mu_{\lambda}(y): \exists a \in A(\{x, z\} \subseteq f(a))\right\}\right) .
\end{gathered}
$$

Similarly we can get

$$
\begin{gathered}
\bigwedge\left\{\max \left(\sup \mu_{\sigma}(y), \sup \mu_{\lambda}(y)\right): \exists a \in A(\{x, z\} \subseteq f(a))\right\} \\
\geq \max \left(\bigwedge\left\{\sup \mu_{\sigma}(y): \exists a \in A(\{x, z\} \subseteq f(a))\right\},\right. \\
\left.\bigwedge\left\{\sup \mu_{\lambda}(y): \exists a \in A(\{x, z\} \subseteq f(a))\right\}\right) .
\end{gathered}
$$

Again since

$$
\begin{aligned}
& \min \left(\inf v_{\sigma}(y), \inf v_{\lambda}(y)\right) \leq \inf v_{\sigma}(y), \\
& \min \left(\inf v_{\sigma}(y), \inf v_{\lambda}(y)\right) \leq \inf \nu_{\lambda}(y)
\end{aligned}
$$

we have

$$
\begin{aligned}
& \bigvee\left\{\min \left(\inf \nu_{\sigma}(y), \inf \nu_{\lambda}(y)\right): \exists a \in A(\{x, y\} \subseteq f(a))\right\} \\
& \quad \leq \bigvee\left\{\inf \nu_{\sigma}(y): \exists a \in A(\{x, z\} \subseteq f(a))\right\} \\
& \bigvee\left\{\min \left(\min \nu_{\sigma}(y), \min \nu_{\lambda}(y)\right): \exists a \in A\{x, y\} \subseteq f(a)\right\} \\
& \quad \leq \bigvee\left\{\inf \nu_{\lambda}(y): \exists a \in A(\{x, z\} \subseteq f(a))\right\} .
\end{aligned}
$$

Consequently,

$$
\begin{gathered}
\bigvee\left\{\min \left(\inf \nu_{\sigma}(y), \inf \nu_{\lambda}(y)\right): \exists a \in A(\{x, y\} \subseteq f(a))\right\} \\
\leq \min \left(\bigvee\left\{\inf \nu_{\sigma}(y): \exists a \in A(\{x, z\} \subseteq f(a))\right\}\right. \\
\left.\bigvee\left\{\inf \nu_{\lambda}(y): \exists a \in A(\{x, y\} \subseteq f(a))\right\}\right) .
\end{gathered}
$$


Similarly we can get

$$
\begin{gathered}
\bigvee\left\{\min \left(\sup \nu_{\sigma}(y), \sup v_{\lambda}(y)\right): \exists a \in A(\{x, y\} \subseteq f(a))\right\} \\
\leq \min \left(\bigvee\left\{\sup \nu_{\sigma}(y): \exists a \in A(\{x, z\} \subseteq f(a))\right\},\right. \\
\left.\bigvee\left\{\sup v_{\lambda}(y): \exists a \in A(\{x, z\} \subseteq f(a))\right\}\right) .
\end{gathered}
$$

Again since

$$
\begin{aligned}
& \max \left(\inf \omega_{\sigma}(y), \inf \omega_{\lambda}(y)\right) \leq \inf \omega_{\sigma}(y), \\
& \max \left(\inf \omega_{\sigma}(y), \inf \omega_{\lambda}(y)\right) \leq \inf \omega_{\lambda}(y),
\end{aligned}
$$

we have

$$
\begin{aligned}
& \bigvee\left\{\min \left(\inf \omega_{\sigma}(y), \inf \omega_{\lambda}(y)\right): \exists a \in A(\{x, y\} \subseteq f(a))\right\} \\
& \quad \leq \bigvee\left\{\inf \omega_{\sigma}(y): \exists a \in A(\{x, z\} \subseteq f(a))\right\}, \\
& \bigvee\left\{\min \left(\inf \omega_{\sigma}(y), \inf \omega_{\lambda}(y)\right): \exists a \in A(\{x, y\} \subseteq f(a))\right\} \\
& \quad \leq \bigvee\left\{\inf \omega_{\lambda}(y): \exists a \in A(\{x, z\} \subseteq f(a))\right\} .
\end{aligned}
$$

Consequently,

$$
\begin{gathered}
\bigvee\left\{\min \left(\inf \omega_{\sigma}(y), \inf \omega_{\lambda}(y)\right): \exists a \in A(\{x, y\} \subseteq f(a))\right\} \\
\leq \min \left(\bigvee\left\{\inf \omega_{\sigma}(y): \exists a \in A(\{x, z\} \subseteq f(a))\right\},\right. \\
\left.\bigvee\left\{\inf \omega_{\lambda}(y): \exists a \in A(\{x, z\} \subseteq f(a))\right\}\right) .
\end{gathered}
$$

Similarly we can get

$$
\begin{gathered}
\bigvee\left\{\min \left(\sup \omega_{\sigma}(y), \sup \omega_{\lambda}(y)\right): \exists a \in A(\{x, z\} \subseteq f(a))\right\} \\
\leq \min \left(\bigvee\left\{\sup \omega_{\sigma}(y): \exists a \in A(\{x, z\} \subseteq f(a))\right\},\right. \\
\left.\bigvee\left\{\sup \omega_{\lambda}(y): \exists a \in A(\{x, z\} \subseteq f(a))\right\}\right) .
\end{gathered}
$$

Using (46)-(55), we get from (43)

$$
\underline{N}_{s}(\sigma) \cup \underline{N}_{s}(\lambda) \subseteq \underline{N}_{s}(\sigma \cap \lambda) .
$$

(8) Proof is similar to (7).

\section{A Multicriteria Group Decision Making Problem}

Soft sets, fuzzy soft sets, intuitionistic fuzzy soft sets, and neutrosophic soft sets have been applied by many authors in solving decision making problems. In this section, we illustrate the use of soft sets and, neutrosophic soft sets, interval-valued neutrosophic soft sets, rough sets, interval-valued neutrosophic soft rough sets, and related notions in object evaluation and group decision making.
Let $U=\left\{o_{1}, o_{2}, o_{3}, \ldots, o_{l}\right\}$ be a set of objects and let $E$ be a set of parameters and $A=\left\{e_{1}, e_{2}, e_{3}, \ldots, e_{l}\right\} \subseteq E$ and let $S=(F, A)$ be a full soft set over $U$. Let us assume that we have an expert group $G=\left\{T_{1}, T_{2}, T_{3}, \ldots, T_{l}\right\}$ consisting of $n$ specialists to evaluate the objects in $U$. Each specialist will examine all the objects in $U$ and will point out his/her evaluation result. Let $X_{i}$ denote the primary evaluation result of the specialist $T_{i}$. It is easy to see that the primary evaluation result of the whole expert group $G$ can be represented as an interval-valued neutrosophic evaluation soft set $S^{*}=\left(F^{*}, G\right)$ over $U$, where $F^{*}: G \rightarrow$ IVNS $^{U}$ is given by $F^{*}\left(T_{i}\right)=$ $X_{i}$, for $i=1,2, \ldots, n$. Now we consider the soft rough interval-valued neutrosophic approximations of the specialist $T_{i}$ 's primary evaluation result $X_{i}$ with respect to the soft approximation space $P=(U, S)$. Then we obtain two other interval-valued neutrosophic soft sets $\underline{S^{*}}=\left(\underline{F^{*}}, G\right)$ and $\overline{S^{*}}=$ $\left(\overline{F^{*}}, G\right)$ over $U$, where $\underline{F^{*}}: G \rightarrow \operatorname{IVNS} \bar{U}$ is given by $\underline{F^{*}}\left(T_{i}\right)=$ $X_{i}$ and $\overline{F^{*}}: G \rightarrow \operatorname{IVNS}^{U}$ is given by $\overline{F^{*}}\left(T_{i}\right)=\overline{X_{i}}$, for $i=$ $\overline{1,2}, \ldots, n$. Here $S^{*}$ can be considered as the evaluation result for the whole expert group $G$ with "low confidence," $\overline{S^{*}}$ can be considered as the evaluation result for the whole expert group $G$ with "high confidence," and $S^{*}$ can be considered as the evaluation result for the whole expert group $G$ with "middle confidence." Let us define two interval-valued neutrosophic sets IVNSet $S^{*}$ and $\overline{\text { IVNSet } S^{*}}$ by

$\underline{\text { IVNSet } S^{*}}$

$$
\begin{aligned}
& =\left\{\left\langleo_{k},\left[\frac{1}{n} \sum_{j=1}^{n} \inf \mu_{F^{*}\left(T_{j}\right)}\left(o_{k}\right), \frac{1}{n} \sum_{j=1}^{n} \sup \underline{\mu_{F^{*}\left(T_{j}\right)}}\left(o_{k}\right)\right],\right.\right. \\
& {\left[\frac{1}{n} \sum_{j=1}^{n} \inf {\underline{F^{*}\left(T_{j}\right)}}\left(o_{k}\right), \frac{1}{n} \sum_{j=1}^{n} \sup {\underline{F^{*}\left(T_{j}\right)}}\left(o_{k}\right)\right] \text {, }} \\
& {\left[\frac{1}{n} \sum_{j=1}^{n} \inf \omega_{\left.\underline{F^{*}\left(T_{j}\right.}\right)}\left(o_{k}\right)\right. \text {, }} \\
& \left.\left.\left.\frac{1}{n} \sum_{j=1}^{n} \sup \omega_{\underline{F}^{*}\left(T_{j}\right)}\left(o_{k}\right)\right]\right\rangle: k=1,2, \ldots, l\right\},
\end{aligned}
$$

IVNSet $S^{*}$

$$
\begin{aligned}
=\left\{\left\langleo_{k},\left[\frac{1}{n} \sum_{j=1}^{n} \inf \mu_{\overline{F^{*}}\left(T_{i}\right)}\left(o_{k}\right), \frac{1}{n} \sum_{j=1}^{n} \sup \mu_{\overline{F^{*}}\left(T_{i}\right)}\left(o_{k}\right)\right],\right.\right. \\
{\left[\frac{1}{n} \sum_{j=1}^{n} \inf \nu_{\overline{F^{*}}\left(T_{i}\right)}\left(o_{k}\right), \frac{1}{n} \sum_{j=1}^{n} \sup \nu_{\overline{F^{*}}\left(T_{i}\right)}\left(o_{k}\right)\right], } \\
{\left[\frac{1}{n} \sum_{j=1}^{n} \inf \omega_{\overline{F^{*}}\left(T_{i}\right)}\left(o_{k}\right),\right.} \\
\left.\left.\left.\frac{1}{n} \sum_{j=1}^{n} \sup \omega_{\overline{F^{*}}\left(T_{i}\right)}\left(o_{k}\right)\right]\right\rangle: k=1,2, \ldots, l\right\} .
\end{aligned}
$$


Now we define another interval-valued neutrosophic set IVNSet $S^{*}$ by

$$
\begin{aligned}
& \text { IVNSet } S^{*} \\
& \begin{aligned}
&\left\{\left\langleo_{k},\left[\frac{1}{n} \sum_{j=1}^{n} \inf \mu_{F^{*}\left(T_{j}\right)}\left(o_{k}\right), \frac{1}{n} \sum_{j=1}^{n} \sup \mu_{F^{*}\left(T_{j}\right)}\left(o_{k}\right)\right],\right.\right. \\
&\left.\qquad \frac{1}{n} \sum_{j=1}^{n} \inf \nu_{F^{*}\left(T_{j}\right)}\left(o_{k}\right), \frac{1}{n} \sum_{j=1}^{n} \sup \nu_{F^{*}\left(T_{j}\right)}\left(o_{k}\right)\right], \\
& {\left[\frac{1}{n} \sum_{j=1}^{n} \inf \omega_{F^{*}\left(T_{j}\right)}\left(o_{k}\right),\right.} \\
&\left.\left.\left.\frac{1}{n} \sum_{j=1}^{n} \sup \omega_{F^{*}\left(T_{j}\right)}\left(o_{k}\right)\right]\right\rangle: k=1,2, \ldots, l\right\} .
\end{aligned}
\end{aligned}
$$

Then clearly,

$$
\underline{\text { IVNSet } S^{*}} \subseteq \text { IVNSet } S^{*} \subseteq \overline{\text { IVNSet } S^{*}} \text {. }
$$

Let $C=\{L$ (low confidence), $M$ (middle confidence), $H$ (high confidence) $\}$ be a set of parameters. Let us consider the interval-valued neutrosophic soft set $S^{* *}=(f, C)$ over $U$, where $f: C \rightarrow$ IVNS $^{U}$ is given by $f(L)=$ IVNSet $^{*}$, $f(M)=$ IVNSet $S^{*}$, and $f(H)=\overline{\text { IVNSet } S^{*}}$. Now given a weighting vector $W=\left(\omega_{L}, \omega_{M}, \omega_{H}\right)$ such that $\omega_{L}, \omega_{M}, \omega_{H} \in$ $[0,1]$, we define $\alpha: U \rightarrow P(U)$ by $\alpha\left(o_{k}\right)=\omega_{L} \diamond s_{f(L)}\left(o_{k}\right)+$ $\omega_{M} \diamond s_{f(M)}\left(o_{k}\right)+\diamond s_{f(H)}\left(o_{k}\right), o_{k} \in U(\diamond$ represents ordinary multiplication), where $s_{f(L)}\left(o_{k}\right)=\left(\inf \mu_{F^{*}\left(T_{j}\right)}+\sup \mu_{F^{*}\left(T_{j}\right)}-\right.$ $\left.\inf \nu_{F^{*}\left(T_{j}\right)} \cdot \sup \nu_{F^{*}\left(T_{j}\right)}-\inf \omega_{\underline{F^{*}}\left(T_{j}\right)} \cdot \sup \omega_{F^{*}\left(T_{j}\right)}\right) / 4$ denotes the score function, the same as $s_{f(M)}\left(o_{k}\right)$ and $s_{f(H)}\left(o_{k}\right)$. Here $\alpha\left(o_{k}\right)$ is called the weighted evaluation value of the alternative $o_{k} \in U$. Finally, we can select the object $o_{p}=\max \left\{\alpha\left(o_{k}\right)\right\}$ : $k=1,2, \ldots, l\}$ as the most preferred alternative.

\section{Algorithm}

(1) Input the original description soft set $(F, A)$.

(2) Construct the interval-valued neutrosophic evaluation soft set $S^{*}=\left(F^{*}, G\right)$.

(3) Compute the soft rough interval-valued neutrosophic approximations and then construct the intervalvalued neutrosophic soft sets $\underline{S^{*}}$ and $\overline{S^{*}}$.

(4) Construct the interval-valued neutrosophic IVNSet $S^{*}$, IVNSet $S^{*}$, and IVNSet $S^{*}$.

(5) Construct the interval-valued neutrosophic soft set $S^{* *}$.

(6) Input the weighting vector $W$ and compute the weighted evaluation values of each alternative $\alpha\left(o_{k}\right)$ of each alternative $o_{k} \in U$.

(7) Select the object $o_{p}$.
TABLE 1

\begin{tabular}{llllll}
\hline & $c_{1}$ & $c_{2}$ & $c_{3}$ & $c_{4}$ & $c_{5}$ \\
\hline$e_{1}$ & 1 & 0 & 1 & 1 & 0 \\
$e_{2}$ & 1 & 1 & 0 & 1 & 0 \\
$e_{3}$ & 0 & 1 & 1 & 1 & 1 \\
$e_{4}$ & 1 & 1 & 0 & 0 & 1 \\
\hline
\end{tabular}

\section{An Illustrative Example}

The following example is adapted from [52] with minor changes.

Let us consider a staff selection problem to fill a position in a private company.

Let $U=\left\{c_{1}, c_{2}, c_{3}, c_{4}, c_{5}\right\}$ be the universe set consisting of five candidates. Let us consider the soft set $S=$ $(F, A)$, which describes the "quality of the candidates," where $A=\left\{e_{1}\right.$ (experience), $e_{2}$ (computer knowledge), $e_{3}$ (young and efficient), $e_{4}$ (good communication skill) $\}$. Let the tabular representation of the soft set $(F, A)$ be as shown in Table 1.

Let $G=\left\{T_{1}, T_{2}, T_{3}, T_{4}\right\}$ be the set of interviewers to judge the quality of the candidate in $U$. Now if $X_{i}$ denotes the primary evaluation result of the interviewer $T_{i}$ (for $i=$ $1,2,3,4)$, then the primary evaluation result of the whole expert group $G$ can be represented as an interval-valued neutrosophic evaluation soft set $S^{*}=\left(F^{*}, G\right)$ over $U$, where $F^{*}: G \rightarrow$ IVNS $^{U}$ is given by $F^{*}\left(T_{i}\right)=X_{i}$ for $i=1,2,3,4$.

Let the tabular representation of $S^{*}$ be given as shown in Table 2.

Let us choose $P=(U, S)$ as the soft interval-valued neutrosophic approximation space. Let us consider the interval-

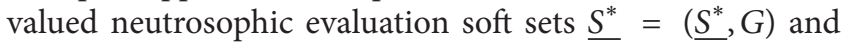
$\overline{S^{*}}=\left(\overline{S^{*}}, G\right)$ over $U$.

Then the tabular representation of these sets is as follows:

$$
\begin{aligned}
& \underline{S^{*}}=\left(\underline{S^{*}}, G\right)(\text { see Table } 3), \\
& \overline{S^{*}}=\left(\overline{S^{*}}, G\right)(\text { see Table } 4) .
\end{aligned}
$$

Here $\underline{S^{*}} \subseteq S^{*} \subseteq \overline{S^{*}}$

$$
\begin{aligned}
& \text { IVNSet } S^{*} \\
& =\left\{\left\langle c_{1},[0.15,0.35],[0.4,0.625],[0.4,0.6]\right\rangle,\right. \\
& \left\langle c_{2},[0.175,0.325],[0.375,0.575],[0.375,0.575]\right\rangle, \\
& \left\langle c_{3},[0.175,0.375],[0.375,0.575],[0.375,0.575]\right\rangle, \\
& \left\langle c_{4},[0.175,0.375],[0.375,0.575],[0.375,0.575]\right\rangle, \\
& \left.\left\langle c_{5},[0.175,0.375][0.375,0.575][0.375,0.6]\right\rangle\right\}
\end{aligned}
$$


TABLE 2

\begin{tabular}{cccccc}
\hline & $c_{1}$ & $c_{2}$ & $c_{3}$ & $c_{4}$ & $\mathcal{c}_{5}$ \\
\hline$T_{1}$ & $([.2, .4],[.4, .5],[.3, .4])$ & $([.6, .7],[.1, .2],[.2, .3])$ & $([.3, .4],[.4, .5],[.2, .5])$ & $([.2, .4],[.4, .6],[.1, .2])$ & $([.3, .6],[.2, .3],[.1, .2])$ \\
$T_{2}$ & $([.1, .3],[.6, .7],[.2, .3])$ & $([.3, .4],[.4, .5],[.2, .4])$ & $([.5, .7],[.1, .2],[.2, .4])$ & $([.7, .8],[.1, .2],[.2, .4])$ & $([.1, .3],[.1, .5],[.2, .5])$ \\
$T_{3}$ & $([.4, .6],[.2, .3],[.4, .5])$ & $([.1, .4],[.2, .4],[.1, .2])$ & $([.2, .5],[.2, .4],[.3, .5])$ & $([.3, .5],[.2, .4],[.4, .6])$ & $([.4, .5],[.2, .5],[.2, .3])$ \\
$T_{4}$ & $([.3, .5],[.3, .4],[.6, .7])$ & $([.5, .6],[.2, .3],[.4, .5])$ & $([.4, .5],[.2, .5],[.1, .2])$ & $([.4, .7],[.1, .2],[.1, .2])$ & $([.6, .8],[.1, .2],[.1, .5])$ \\
\hline
\end{tabular}

TABLE 3

\begin{tabular}{cccccc}
\hline & $c_{1}$ & $c_{2}$ & $c_{3}$ & $c_{4}$ & $c_{5}$ \\
\hline$T_{1}$ & $([.2, .4],[.4, .6],[.4, .5])$ & $([.2, .3],[.4, .6],[.3, .5])$ & $([.2, .3],[.4, .6],[.3, .5])$ & $([.2, .3],[.4, .6],[.3, .5])$ & $([.2, .3],[.4, .6],[.3, .5])$ \\
$T_{2}$ & $([.1, .2],[.6, .8],[.2, .5])$ & $([.1, .3],[.6, .7],[.2, .5])$ & $([.1,3],[.6, .7],[.2, .5])$ & $([.1, .3],[.6, .7],[.2, .5])$ & $([.1, .3],[.6, .7],[.2, .6])$ \\
$T_{3}$ & $([.1, .4],[.2, .5],[.4, .6])$ & $([.1, .2],[.2, .5],[.4, .6])$ & $([.1, .4],[.2, .5],[.4, .6])$ & $([.1, .4],[.2, .5],[.4, .6])$ & $([.1, .4],[.2, .6],[.4, .6])$ \\
$T_{4}$ & $([.2, .4],[.4, .5],[.6, .8])$ & $([.3, .5],[.3, .5],[.6, .7])$ & $([.3, .5],[.3, .5],[.6, .7])$ & $([.3, .5],[.3, .5],[.6, .7])$ & $([.3, .5],[.3, .5],[.6, .7])$ \\
\hline
\end{tabular}

TABLE 4

\begin{tabular}{cccccc}
\hline & $c_{1}$ & $c_{2}$ & $\mathcal{c}_{3}$ & $c_{4}$ & $c_{5}$ \\
\hline$T_{1}$ & $([.6, .7],[.1, .2],[.3, .2])$ & $([.6, .8],[.1, .2],[.1, .2])$ & $([.6, .7],[.1, .2],[.1, .2])$ & $([.6, .7],[.1, .2],[.1, .2])$ & $([.6, .7],[.1, .2],[.1, .2])$ \\
$T_{2}$ & $([.7, .8],[.1, .2],[.2, .4])$ & $([.7, .8],[.1, .2],[.2, .3])$ & $([.7, .8],[.1, .2],[.2, .3])$ & $([.5, .7],[.1, .2],[.2, .3])$ & $([.7, .8],[.1, .2],[.2, .3])$ \\
$T_{3}$ & $([.4, .7],[.2, .3],[.3, .7])$ & $([.4, .6],[.2, .3],[.1, .2])$ & $([.4, .6],[.2, .3],[.1, .2])$ & $([.4, .6],[.2, .3],[.1, .2])$ & $([.4, .6],[.2, .3],[.1, .2])$ \\
$T_{4}$ & $([.6, .8],[.1, .2],[.1, .3])$ & $([.6, .8],[.1, .2],[.1, .2])$ & $([.6, .8],[.1, .2],[.1, .2])$ & $([.6, .8],[.1, .2],[.1, .2])$ & $([.5, .7],[.1, .2],[.1, .2])$ \\
\hline
\end{tabular}

$$
\begin{aligned}
& \hline \text { IVNSet } S^{*} \\
&=\left\{\left\langle c_{1},[0.575,0.75],[0.125,0.225],[0.125,0.225]\right\rangle,\right. \\
&\left\langle c_{2},[0.575,0.75],[0.125,0.225],[0.125,0.225]\right\rangle, \\
&\left\langle c_{3},[0.575,0.725],[0.125,0.225],[0.125,0.225]\right\rangle, \\
&\left\langle c_{4},[0.525,0.700],[0.125,0.225],[0.125,0.225]\right\rangle, \\
&\left.\left\langle c_{5},[0.55,0.700],[0.125,0.225],[0.125,0.225]\right\rangle\right\} .
\end{aligned}
$$

\section{IVNSet $S^{*}$}

$$
\begin{aligned}
= & \left\{\left\langle c_{1},[0.25,0.45],[375,0.475],[0.375,0.475]\right\rangle,\right. \\
& \left\langle c_{2},[0.375,0.525],[0.225,0.35],[0.225,0.35]\right\rangle, \\
& \left\langle c_{3},[0.350,0.525],[0.2,0.4],[0.2,0.4]\right\rangle, \\
& \left\langle c_{4},[0.4,0.6],[0.20,0.35],[0.2,0.35]\right\rangle, \\
& \left.\left\langle c_{5},[0.35,0.55],[0.15,0.375],[0.15,0.375]\right\rangle\right\} .
\end{aligned}
$$

Here IVNSet $S^{*} \subseteq$ IVNSet $S^{*} \subseteq \overline{\text { IVNSet } S^{*}}$. Let $C=\{L$ (low convidence), $M$ (middle confidence), $H$ (high confidence) $\}$ be a set of parameters. Let us consider the interval-valued neutrosophic soft set $S^{* *}=(f, C)$ over $U$, where $f: C \longrightarrow$ IVNS $^{U}$ is given by $f(L)=$ IVNSet $S^{*}, f(M)=$ IVNSet $S^{*}$,

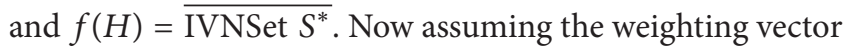

$W=\left(\omega_{L}, \omega_{M}, \omega_{H}\right)$ such that $\omega_{L}=0.7, \omega_{M}=0.6$, and $\omega_{H}=$ 0.8 , we have

$$
\begin{aligned}
\alpha\left(c_{1}\right) & =0.7 \diamond 0.0025+0.6 \diamond 0.0859+0.8 \diamond 0.3171 \\
& =0.3070 \\
\alpha\left(c_{2}\right) & =0.7 \diamond 0.0171+0.6 \diamond 0.1856+0.8 \diamond 0.3171 \\
& =0.3770 \\
\alpha\left(c_{3}\right) & =0.7 \diamond 0.0171+0.6 \diamond 0.1787+0.8 \diamond 0.3109 \\
& =0.3679 \\
\alpha\left(c_{4}\right) & =0.7 \diamond 0.0171+0.6 \diamond 0.2150+0.8 \diamond 0.2921 \\
& =0.3747 \\
\alpha\left(c_{5}\right) & =0.7 \diamond 0.0273+0.6 \diamond 0.1968+0.8 \diamond 0.2023 \\
& =0.2991 .
\end{aligned}
$$

Since $\max \left(\alpha\left(c_{1}\right), \alpha\left(c_{2}\right), \alpha\left(c_{3}\right), \alpha\left(c_{4}\right), \alpha\left(c_{5}\right)\right\}=0.3770$, so the candidate $c_{2}$ will be selected as the most preferred alternative.

\section{Conclusions}

In this paper we have defined, for the first time, the notion of interval-valued neutrosophic soft rough sets which is a combination of interval-valued neutrosophic soft sets and rough sets. We have studied some of their basic properties. Thus our work is a generalization of IVIF-soft rough sets and intuitionistic fuzzy soft rough sets. We hope that this paper will promote the future study on interval-valued neutrosophic soft 
rough sets to carry out a general framework for their application in practical life.

\section{Conflict of Interests}

The authors declare that there is no conflict of interests regarding the publication of this paper.

\section{Acknowledgment}

The authors are very grateful to the anonymous referees for their insightful and constructive comments and suggestions, which have been very helpful in improving the paper.

\section{References}

[1] F. Smarandache, A Unifying Field in Logics. Neutrosophy: Neutrosophic Probability, Set and Logic, American Research Press, Rehoboth, NM, USA, 1999.

[2] L. A. Zadeh, "Fuzzy sets," Information and Computation, vol. 8, pp. 338-353, 1965.

[3] K. T. Atanassov, "Intuitionistic fuzzy sets," Fuzzy Sets and Systems, vol. 20, no. 1, pp. 87-96, 1986.

[4] I. B. Turksen, "Interval valued fuzzy sets based on normal forms," Fuzzy Sets and Systems, vol. 20, no. 2, pp. 191-210, 1968.

[5] M. Bhowmik and M. Pal, "Intuitionistic neutrosophic set," Journal of Information and Computing Science, vol. 4, no. 2, pp. 142152, 2009.

[6] M. Bhowmik and M. Pal, "Intuitionistic neutrosophic set relations and some of its properties," Journal of Information and Computing Science, vol. 5, no. 3, pp. 183-192, 2010.

[7] A. A. Salama and S. A. Alblowi, "Generalized neutrosophic set and generalized neutrosophic topological spaces," Computer Science and Engineering, vol. 2, no. 7, pp. 129-132, 2012.

[8] H. Wang, F. Smarandache, Y. Q. Zhang, and R. Sunderraman, "Single valued neutrosophic sets," Multispace and Multistructure, vol. 4, pp. 410-413, 2010.

[9] H. Wang, F. Smarandache, Y.-Q. Zhang, and R. Sunderraman, Interval Neutrosophic Sets and Logic: Theory and Applications in Computing, Hexis, Phoenix, Ariz, USA, 2005.

[10] K. Georgiev, "A simplification of the neutrosophic sets. Neutrosophic logic and intuitionistic fuzzy sets," in Proceedings of the 9th International Conference on IFSs, vol. 11, no. 2 of NIFS, pp. 28-31, Sofia, Bulgaria, May 2005.

[11] J. Ye, "Single valued netrosophiqc minimum spanning tree and its clustering method," Journal of Intelligent System, vol. 23, no. 3, pp. 311-324, 2014.

[12] J. Ye, "Similarity measures between interval neutrosophic sets and their applications in multicriteria decision-making," Journal of Intelligent \& Fuzzy Systems, vol. 26, no. 1, pp. 165-172, 2014.

[13] P. Majumdar and S. K. Samant, "On similarity and entropy of neutrosophic sets," Journal of Intelligent and Fuzzy Systems, vol. 26, no. 3, pp. 1245-1252, 2013.

[14] S. Broumi and F. Smarandache, "Several similarity measures of neutrosophic sets," Neutrosophic Sets and Systems, vol. 1, pp. 5462, 2013.

[15] S. Broumi and F. Smarandache, "Correlation coefficient of interval neutrosophic set," Applied Mechanics and Materials, vol. 436, pp. 511-517, 2013.
[16] S. Broumi and F. Smarandache, "New operations on interval neutrosophic sets," Journal of New Theory, no. 1, pp. 24-37, 2015, http://www.newtheory.org/.

[17] P. Chi and L. Peide, "An extended TOPSIS method for the multiple attribute decision making problems based on interval neutrosophic," Neutrosophic Sets and Systems, vol. 1, pp. 63-70, 2013.

[18] D. Molodtsov, "Soft set theory-first results," Computers \& Mathematics with Applications, vol. 37, no. 4-5, pp. 19-31, 1999.

[19] I. Deli, "Interval-valued neutrosophic soft sets and its decision making," Journal of Multiple-Valued Logic and Soft Computing. (Submitted).

[20] P. K. Maji, R. Biswas, and A. R. Roy, "Fuzzy soft sets," Journal of Fuzzy Mathematics, vol. 9, no. 3, pp. 589-602, 2001.

[21] P. K. Maji, R. Biswas, and A. R. Roy, "Intuitionistic fuzzy soft sets," Journal of Fuzzy Mathematics, vol. 9, no. 3, pp. 677-692, 2001.

[22] P. K. Maji, "Neutrosophic soft set," Annals of Fuzzy Mathematics and Informatics, vol. 5, no. 1, pp. 157-168, 2013.

[23] S. Broumi and F. Smarandache, "Intuitionistic neutrosophic soft set," Journal of Information and Computing Science, vol. 8, no. 2, pp. 130-140, 2013.

[24] Z. Pawlak, "Rough sets," International Journal of Computer and Information Sciences, vol. 11, no. 5, pp. 341-356, 1982.

[25] D. Dubios and H. Prade, "Rough fuzzy sets and fuzzy rough sets," International Journal of General Systems, vol. 17, pp. 191208, 1990.

[26] Z. Gong, B. Sun, and D. Chen, "Rough set theory for the interval-valued fuzzy information systems," Information Sciences, vol. 178, no. 8, pp. 1968-1985, 2008.

[27] B. Sun, Z. Gong, and D. Chen, "Fuzzy rough set theory for the interval-valued fuzzy information systems," Information Sciences, vol. 178, no. 13, pp. 2794-2815, 2008.

[28] W.-Z. Wu, J.-S. Mi, and W.-X. Zhang, "Generalized fuzzy rough sets," Information Sciences, vol. 151, pp. 263-282, 2003.

[29] Z. Zhang, "On interval type-2 rough fuzzy sets," KnowledgeBased Systems, vol. 35, pp. 1-13, 2012.

[30] J.-S. Mi, Y. Leung, H.-Y. Zhao, and T. Feng, "Generalized fuzzy rough sets determined by a triangular norm," Information Sciences, vol. 178, no. 16, pp. 3203-3213, 2008.

[31] K. V. Thomas and L. S. Nair, "Rough intutionistic fuzzy sets in a lattice," International Mathematical Forum, vol. 6, no. 27, pp. 1327-1335, 2011.

[32] Q. He, C. Wu, D. Chen, and S. Zhao, "Fuzzy rough set based attribute reduction for information systems with fuzzy decisions," Knowledge-Based Systems, vol. 24, no. 5, pp. 689-696, 2011.

[33] F. Min and W. Zhu, "Attribute reduction of data with error ranges and test costs," Information Sciences, vol. 211, pp. 48-67, 2012.

[34] F. Min, H. He, Y. Qian, and W. Zhu, “Test-cost-sensitive attribute reduction," Information Sciences, vol. 181, no. 22, pp. 49284942, 2011.

[35] Y. Yao and Y. Zhao, "Attribute reduction in decision-theoretic rough set models," Information Sciences, vol. 178, no. 17, pp. 3356-3373, 2008.

[36] M. Dash and H. Liu, "Consistency-based search in feature selection," Artificial Intelligence, vol. 151, no. 1-2, pp. 155-176, 2003. 
[37] Q. Hu, D. Yu, J. Liu, and C. Wu, "Neighborhood rough set based heterogeneous feature subset selection," Information Sciences, vol. 178, no. 18, pp. 3577-3594, 2008.

[38] T.-L. (Bill) Tseng and C.-C. Huang, "Rough set-based approach to feature selection in customer relationship management," Omega, vol. 35, no. 4, pp. 365-383, 2007.

[39] B. Baesens, R. Setiono, C. Mues, and J. Vanthienen, "Using neural network rule extraction and decision tables for creditrisk evaluation," Management Science, vol. 49, no. 3, pp. 312-329, 2003.

[40] R. Cruz-Cano, M.-L. T. Lee, and M.-Y. Leung, "Logic minimization and rule extraction for identification of functional sites in molecular sequences," BioData Mining, vol. 5, no. 1, article 10, 2012.

[41] Y. Du, Q. Hu, P. Zhu, and P. Ma, "Rule learning for classification based on neighborhood covering reduction," Information Sciences, vol. 181, no. 24, pp. 5457-5467, 2011.

[42] X. Wang, E. C. Tsang, S. Zhao, D. Chen, and D. S. Yeung, "Learning fuzzy rules from fuzzy samples based on rough set technique," Information Sciences, vol. 177, no. 20, pp. 4493-4514, 2007.

[43] F. Feng, "Soft rough sets applied to multicriteria group decision making," Annals of Fuzzy Mathematics and Informatics, vol. 2, no. 1, pp. 69-80, 2011.

[44] S. Broumi and F. Smarandache, "Lower and upper soft interval valued neutrosophic rough approximations of an IVNSSrelation," in Proceedings of the SISOM \& ACOUSTICS, pp. 1-8, 2014.

[45] F. Feng, C. Li, B. Davvaz, and M. I. Ali, "Soft sets combined with fuzzy sets and rough sets: a tentative approach," Soft Computing, vol. 14, no. 9, pp. 899-911, 2010.

[46] M. Shabir, M. I. Ali, and T. Shaheen, "Another approach to soft rough sets," Knowledge-Based Systems, vol. 40, pp. 72-80, 2013.

[47] H. Zhang, L. Shu, and S. Liao, "Intuitionistic fuzzy soft rough set and its application in decision making," Abstract and Applied Analysis, vol. 2014, Article ID 287314, 13 pages, 2014.

[48] A. Saha and A. Mukherjee, "Soft interval-valued intuitionistic fuzzy rough sets," Annals of Fuzzy Mathematics and Informatics, vol. 9, no. 2, pp. 279-292, 2015.

[49] S. Broumi, F. Smarandache, and M. Dhar, "Rough neutrosophic sets," Italian Journal of Pure and Applied Mathematics, no. 32, pp. 493-502, 2014.

[50] A. A. Salama and S. Broumi, "Roughness of neutrosophic sets," Elixir Applied Mathematics, vol. 74, pp. 26833-26837, 2014.

[51] A. Mukherjee, A. Saha, and A. K. Das, "Interval valued intuitionistic fuzzy soft set relations," Annals of Fuzzy Mathematics and Informatics, vol. 7, no. 4, pp. 563-577, 2014.

[52] A. Mukherjee and A. Saha, "Interval-valued intuitionistic fuzzy soft rough sets," Annals of Fuzzy Mathematics and Informatics, vol. 5, no. 3, pp. 533-547, 2013. 


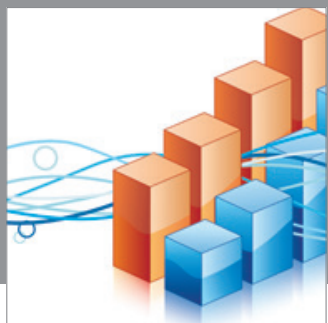

Advances in

Operations Research

mansans

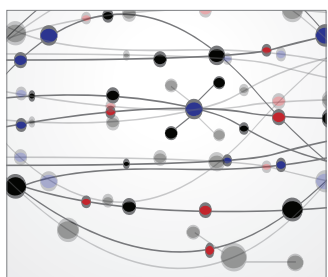

The Scientific World Journal
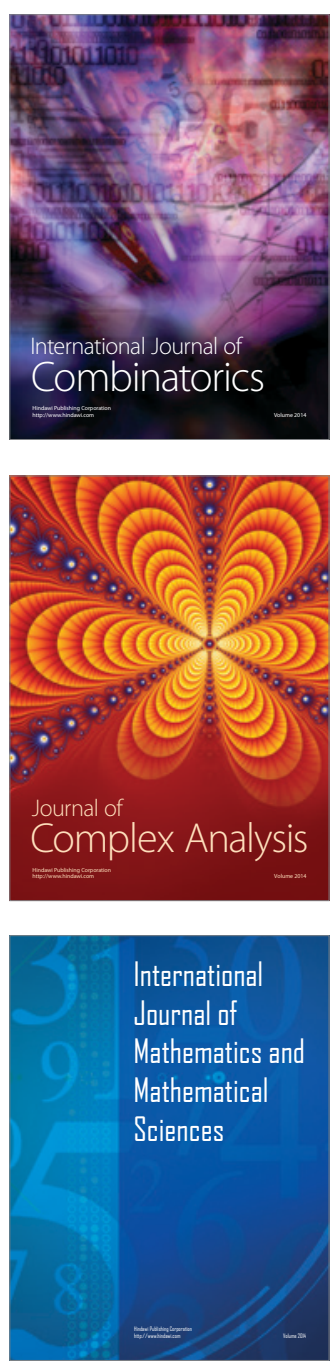
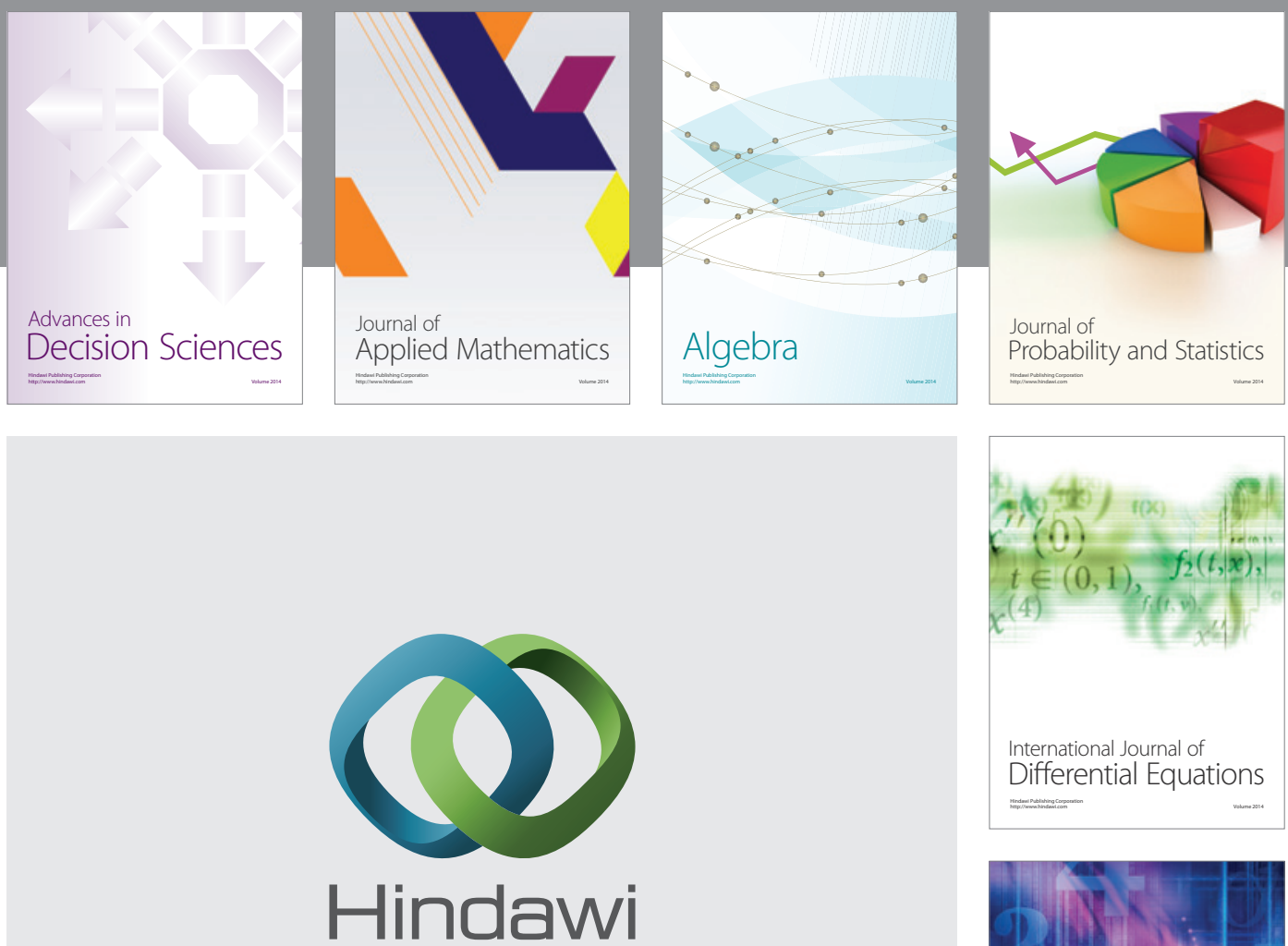

Submit your manuscripts at http://www.hindawi.com
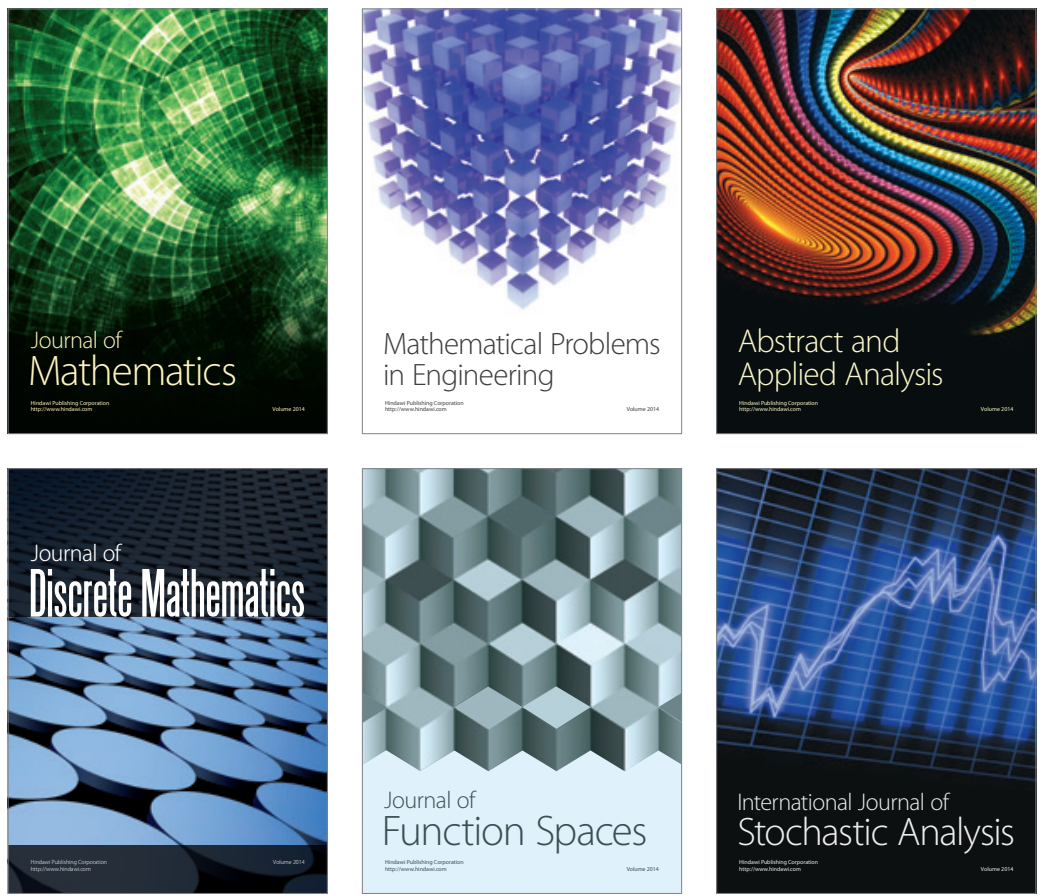

Journal of

Function Spaces

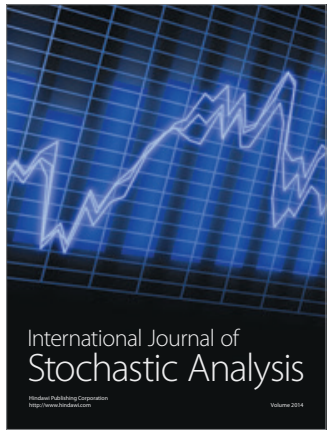

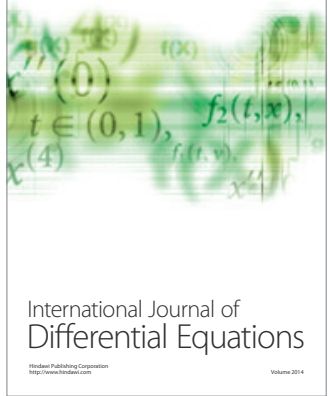
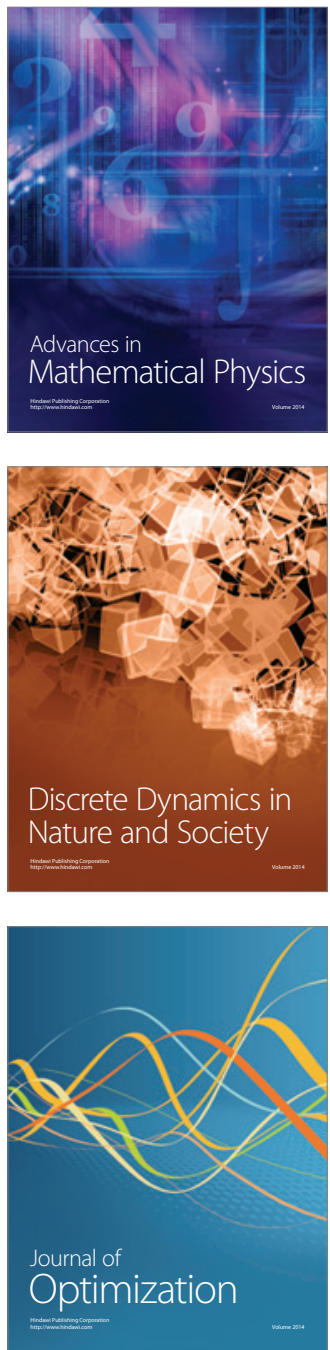